\title{
Simulating Impedance Spectra from a Mechanistic Point of View: Theory and Simulations
}

\author{
Elisabet Ahlberg* and Helge Anderson
}

Department of Inorganic Chemistry, Chalmers University of Technology and University of Göteborg, S-412 96 Göteborg, Sweden

\begin{abstract}
Ahlberg, E. and Anderson, H., 1992. Simulating Impedance Spectra from a Mechanistic Point of View: Theory and Simulations. - Acta Chem. Scand. 46: 1-14.

The faradaic impedance for complex mechanisms with one, two or three adsorbed intermediates has been developed using an analytical solution. A number of mechanisms have been simulated and some general characteristics are presented. For a mechanism with two or more adsorbed intermediates there is no unambiguous relationship between the experimental time constants, obtained from the spectrum, and the time constants found from the mechanistic model. It is also shown that for mechanisms involving pure chemical steps the $\ln (10) R_{\mathrm{p}} I_{\mathrm{ss}}$ product cannot always be used as a Tafel value.
\end{abstract}

The investigation of complex heterogeneous electrochemical processes often requires transient techniques. Depending on the technique used, the response of the transient is analysed in either the time or the frequency domain. Impedance spectroscopy (IS) is an example of frequency response analysis which has been shown to be useful in both applied and fundamental electrochemistry, as well as in other disciplines. ${ }^{1,2}$ The power of the technique is its ability to distinguish between processes with different time constants at the interface. This is especially important for the investigation of heterogeneous electrochemical reaction mechanisms, where adsorption and desorption processes are likely to occur.

In order to extract mechanistic information from IS it is important to measure the potential dependence of the impedance and the steady-state current in the potential region of interest. Since there are no a priori methods to determine a reaction mechanism from impedance and polarization data, a model has to be proposed and tested for the various experimental conditions. In IS, chemical models as well as purely electrical models have been used to interpret reaction mechanisms. The electrical models are necessary to use in more complicated systems, where details of the individual steps in the reaction mechanism are unattainable. However, with a chemical model a more fundamental coupling between the detailed mechanism and experimental data is obtainable. An important part of the IS investigation is therefore the derivation of the impedance function for a certain reaction mechanism. This function is usually very complicated, even for simple mechanisms with few elementary steps involved. Thus it is very difficult to predict the impedance behaviour without any calculations. Although the number of studies in which
IS has been used has grown quickly during the last decade, not much has been written about how the impedance characteristics are related to different types of reaction models; for example, how the impedance is affected by a change in the rate-determining step or the presence of pure chemical or catalytical steps.

We have developed a computer program for the calculation of the steady-state polarization curves and the impedance spectra for a number of reaction mechanisms. By varying parameters such as the rate constants and the potential, we are able to study the polarization and impedance characteristics of different reaction models. With the zinc dissolution reaction in mind a number of reaction mechanisms have been simulated.

In a subsequent paper ${ }^{3}$ the details of the anodic dissolution of zinc in slightly acidic solution will be presented.

\section{Derivation of the impedance}

The impedance function is a special case of the more general transfer function used in system analysis. If the pertubation signal $x(t)$ is a sine wave [eqn. (1)], the response $y(t)$ is also a sine wave [eqn. (2)], with an angular frequency $\omega$ and a phase shift $\varphi$.

$$
\begin{aligned}
& x(t)=X \sin \omega t \\
& y(t)=Y \sin (\omega t+\varphi)
\end{aligned}
$$

The transfer function may then be defined by eqn. (3), where $|H(\omega)|=Y / X$.

$$
H(\omega)=|H(\omega)| \exp (\mathrm{i} \varphi)
$$

\footnotetext{
${ }^{*}$ To whom correspondence should be addressed.
} 
If $x(t)$ is a voltage and $y(t)$ is a current then $H(\omega)$ is an admittance value, the inverse of the impedance value. The transfer function is only valid when the system fulfils the following conditions: (1) causality (the response of the system must be caused by the input signal only); (2) stability (the system must return to its initial steady state after the perturbation); (3) linearity (the system must show a linear response to the pertubation) and (4) infinity (the impedance must be finite when the frequency goes towards zero and infinity and at all intermediate frequencies). These conditions can be tested by the use of the KramersKronig transformation on a set of data. The KramersKronig transformation has been treated in detail for electrochemical purposes. ${ }^{4-7}$

The measured quantity in IS is usually the AC current. In order to simulate the impedance spectra we must derive how this current responds to the voltage pertubation for a given reaction mechanism. There are several ways to do this. We have used the method outlined by Epelboin et al. ${ }^{8}$ However, this method rests on some additional assumptions that have to be made: (1) the reactions follow Tafel kinetics; (2) the adsorption of intermediates follow the Langmuir isotherm and (3) charge transfer and adsorption/ desorption are the only processes occuring at the interface.

Under these conditions the faradaic impedance is defined by eqn. (4), where $\Delta E=E-E_{\mathrm{ss}}$ and $\Delta I_{\mathrm{f}}=I_{\mathrm{f}}-I_{\mathrm{ss}}$. If

$\frac{1}{Z_{\mathrm{f}}}=\frac{\Delta I_{\mathrm{f}}}{\Delta E}$

the pertubation signal is a sine wave we may express it by eqn. (5).

$\Delta E=|\Delta E| \exp (\mathrm{i} \omega t)$

The faradaic current is a function of potential but may also be dependent on other state variables, such as the concentration of adsorbed intermediates. The deviation of the faradaic current from its steady-state value is therefore obtained by taking the total differential of the current with respect to all state variables depending on $E$. If we use a small sine wave $(<10 \mathrm{mV})$ to pertubate the system we can approximate a linear relation between the current and the potential. The differential may then be obtained by using terms of first order only, as in eqn. (6), where $\theta_{i}$ is the

$\Delta I_{\mathrm{f}}=\frac{\partial I_{\mathrm{f}}}{\partial E} \Delta E+\sum_{i} \frac{\partial I_{\mathrm{f}}}{\partial \theta_{i}} \Delta \theta_{i}$

fraction of the electrode covered by the adsorbed species $i$. Division by $\Delta E$ gives the faradaic admittance $Y_{\mathrm{f}}=1 / Z_{\mathrm{f}}$, as in eqn. (7), in which quantities are derived from the charge-

$\frac{1}{Z_{\mathrm{f}}}=\frac{\partial I_{\mathrm{f}}}{\partial E}+\sum_{i} \frac{\partial I_{\mathrm{f}}}{\partial \theta_{i}} \frac{\Delta \theta_{i}}{\Delta E}$

and mass-balance equations under steady-state conditions.
The charge balance is given by eqn. (8), where $K_{n}$ is the

$I=F\left[\sum_{n}\left(K_{n}-K_{-n}\right)\right]$

normalized rate constant (in mol $\mathrm{cm}^{-2} \mathrm{~s}^{-1}$ ), which is the product of the rate constant $k_{n}$ and the concentration of the reaction species. The rate constants are assumed to vary exponentially with the potential as in eqn. (9) (anodic reactions) and eqn. (10) (cathodic reactions).

$$
\begin{aligned}
& k_{n}=k_{n}^{\circ} \exp \left(\frac{(1-\alpha) z F}{R T}\left(E-E^{\circ}\right)\right) \\
& k_{-n}=k_{n}^{\circ} \exp \left(\frac{-\alpha z F}{R T}\left(E-E^{\circ}\right)\right)
\end{aligned}
$$

The mass balance is written as eqn. (11), where $v_{n, \theta_{i}}$ is the

$\beta_{i} \frac{\mathrm{d} \theta_{i}}{\mathrm{~d} t}=\sum_{n} v_{n, \theta_{i}}$

rate of formation or disappearance of $\theta_{i}$ caused by the $n$th step (desorption rates taking negative values).

$\partial I / \partial E$ and $\partial I / \partial \theta_{i}$ are obtained from the charge balance equation. The steady-state solution, $\theta_{i, \mathrm{ss}}$, is given from the mass balance equation with $\mathrm{d} \theta_{i} / \mathrm{d} t=0 . \Delta \theta_{i} / \Delta E$ can then be obtained by noting that for a small sine-wave pertubation of the potential, the total differential of $\beta_{i} \mathrm{~d} \theta_{i} / \mathrm{d} t$ can be written as $i \omega \beta_{i} \Delta \theta_{i}$, where $\Delta \theta_{i}$ is the time variation of $\theta_{i}$ caused by the perturbation signal $\left[\Delta \theta_{i}=\left|\Delta \theta_{i}\right| \exp (i \omega t)\right]$. If all $\beta_{i}$ are equal to $\beta$ we obtain the set of linear equations (12)-(14).

$$
\begin{aligned}
& i \omega \beta \Delta \theta_{1}=\frac{\partial\left(\frac{\mathrm{d} \theta_{1}}{\mathrm{~d} t}\right)}{\partial E} \Delta E+\sum_{i=1}^{m} \frac{\partial\left(\frac{\mathrm{d} \theta_{1}}{\mathrm{~d} t}\right)}{\partial \theta_{i}} \Delta \theta_{i} \\
& i \omega \beta \Delta \theta_{2}=\frac{\partial\left(\frac{\mathrm{d} \theta_{2}}{\mathrm{~d} t}\right)}{\partial E} \Delta E+\sum_{i=1}^{m} \frac{\partial\left(\frac{\mathrm{d} \theta_{2}}{\mathrm{~d} t}\right)}{\partial \theta_{i}} \Delta \theta_{i} \\
& i \omega \beta \Delta \theta_{m}=\frac{\partial\left(\frac{\mathrm{d} \theta_{m}}{\mathrm{~d} t}\right)}{\partial E} \Delta E+\sum_{i=1}^{m} \frac{\partial\left(\frac{\mathrm{d} \theta_{m}}{\mathrm{~d} t}\right)}{\partial \theta_{i}} \Delta \theta_{i}
\end{aligned}
$$

After rearrangement and division by $\Delta E$ the system of equations can be solved for $\Delta \theta_{i} / \Delta E$. The solution of the equation system when the number of state variables is one or two has been given by Cao. ${ }^{9,10}$ We have generalized the solution to include also three variables. The solutions are given by eqns. (15)-(20). 
$m=1$ :

$\frac{\Delta \theta_{1}}{\Delta E}=\frac{a_{1}}{T+\mathrm{i} \omega \beta}$

$m=2$ :

$\frac{\Delta \theta_{1}}{\Delta E}=\frac{-J_{22} a_{1}+J_{12} a_{2}+i \omega \beta a_{1}}{D-\omega^{2} \beta^{2}+i \omega \beta T}$

$\frac{\Delta \theta_{2}}{\Delta E}=\frac{-J_{21} a_{1}+J_{11} a_{2}+\mathrm{i} \omega \beta a_{2}}{D-\omega^{2} \beta^{2}+\mathrm{i} \omega \beta T}$

$m=3:$

$\frac{\Delta \theta_{1}}{\Delta E}=$

$\frac{-L_{11} a_{1}+L_{21} a_{2}-L_{31} a_{3}+\mathrm{i} \omega \beta\left[\left(J_{22}+J_{33}\right) a_{1}-J_{12} a_{2}+J_{13} a_{3}\right]+\omega^{2} \beta^{2} a_{1}}{D-\mathrm{i} \omega \beta\left(L_{11}+L_{22}+L_{33}\right)-\omega^{2} \beta^{2} T+\mathrm{i} \omega^{3} \beta^{3}}$

$\frac{\Delta \theta_{2}}{\Delta E}=$

$\frac{L_{12} a_{1}-L_{22} a_{2}+L_{32} a_{3}+\mathrm{i} \omega \beta\left[-J_{21} a_{1}+\left(J_{11}+J_{33}\right) a_{2}-J_{23} a_{3}\right]+\omega^{2} \beta^{2} a_{2}}{D-\mathrm{i} \omega \beta\left(L_{11}+L_{22}+L_{33}\right)-\omega^{2} \beta^{2} T+\mathrm{i} \omega^{3} \beta^{3}}$

$\frac{\Delta \theta_{3}}{\Delta E}=$

$\frac{-L_{13} a_{1}+L_{23} a_{2}-L_{33} a_{3}+\mathrm{i} \omega \beta\left[J_{31} a_{1}-J_{32} a_{2}+\left(J_{11}+J_{22}\right) a_{3}\right]+\omega^{2} \beta^{2} a_{3}}{D-i \omega \beta\left(L_{11}+L_{22}+L_{33}\right)-\omega^{2} \beta^{2} T+\mathrm{i} \omega^{3} \beta^{3}}$

The parameters $a_{i}, J_{i j}, L_{i j}, D$ and $T$ are defined in Appendix I. With an additional set of parameters, $A, B, C$ and $S$ (also defined in Appendix I) the faradaic impedance, eqn. (7), can be written as eqns. (21)-(23), where $1 / R_{\mathrm{t}}=$

$m=1:$

$\frac{1}{Z_{\mathrm{f}}}=\frac{1}{R_{\mathrm{t}}}+\frac{B}{T+\mathrm{i} \omega \beta}$

$m=2$ :

$\frac{1}{Z_{\mathrm{f}}}=\frac{1}{R_{\mathrm{t}}}+\frac{A+\mathrm{i} \omega \beta B}{D-\omega^{2} \beta^{2}+\mathrm{i} \omega \beta T}$

$m=3$

$\frac{1}{Z_{\mathrm{f}}}=\frac{1}{R_{\mathrm{t}}}+\frac{A^{\prime}+\mathrm{i} \omega \beta C+\omega^{2} \beta^{2} B}{D-\mathrm{i} \omega \beta S-\omega^{2} \beta^{2} T+\mathrm{i} \omega^{3} \beta^{3}}$

$\partial I / \partial E$. The interfacial impedance, $Z_{\mathrm{I}}$, is obtained by adding the double-layer capacitance, $C_{\mathrm{dl}}$, in parallel with the faradaic impedance eqn. (24) or (25). An example of this
$\frac{1}{Z_{\mathrm{l}}}=\frac{1}{Z_{\mathrm{f}}}+\mathrm{i} \omega C_{\mathrm{dl}}$

$Z_{\mathrm{I}}=\frac{Z_{\mathrm{f}}}{1+\mathrm{i} \omega C_{\mathrm{dl}} Z_{\mathrm{f}}}$

derivation is given in Appendix II, where a mechanism with two adsorbed intermediates is treated in detail.

\section{Results}

In the following the characteristics of some mechanisms will be reported. The aim is to illustrate the influence of an autocatalytic step and/or a chemical step on the impedance and polarization behaviour. The mechanisms have been chosen with the zinc dissolution reaction in mind. The impedance functions are, as mentioned, often very complex, and in most cases several parameters are free to vary. It is therefore not possible to view the whole impedance surface. We have chosen to present the results in threedimensional plots where the $x z$-plane corresponds to the $Z^{\prime} Z^{\prime \prime}$-plane and the $y$-axis is the potential. Each diagram shows the resulting spectra for one set of rate constants at five potentials, 10, 20, 30, 40 and $50 \mathrm{mV}$, respectively. The rate constants used in the simulations were chosen so that one step in the reaction mechanism was made slow compared to the others. In some cases other combinations were used in order to illustrate certain features. The combinations of rate constants are listed in Tables 1-4. Other parameters of the corresponding mechanisms are listed in Table $5 . E^{\circ}$ is in all simulations equal to $0 \mathrm{mV}$.

EE-mechanism:

$\mathrm{Zn} \underset{k_{-1}}{\stackrel{k_{1}}{\rightleftharpoons}} \mathrm{Zn}_{\mathrm{ad}}^{+} \stackrel{k_{2}}{\longrightarrow} \mathrm{Zn}_{\mathrm{aq}}^{2+}$

Table 1. Rate constants (in mol $\mathrm{cm}^{-2} \mathrm{~s}^{-1}$ ) used in the simulation of the EE-mechanism and the catalytic EE-mechanism.

\begin{tabular}{llll}
\hline Fig. & $K_{1}^{\circ}$ & $K_{-1}^{\circ}$ & $K_{2}^{\circ}$ \\
\hline $1(\mathrm{~b}) / 2(\mathrm{~b})$ & $10^{-7}$ & $10^{-7}$ & $10^{-8}$ \\
$1(\mathrm{c}) / 2(\mathrm{c})$ & $10^{-8}$ & $10^{-8}$ & $4 \times 10^{-8}$ \\
$1(\mathrm{~d}) / 2(\mathrm{~d})$ & $10^{-8}$ & $10^{-8}$ & $10^{-7}$ \\
$1(\mathrm{e}) / 2(\mathrm{e})$ & $10^{-8}$ & $10^{-8}$ & $10^{-8}$ \\
\hline
\end{tabular}

Table 2. Rate constants (in $\mathrm{mol} \mathrm{cm} \mathrm{cm}^{-2} \mathrm{~s}^{-1}$ ) used in the simulation of the EEC-mechanism.

\begin{tabular}{llllll}
\hline Fig. & $K_{1}^{\circ}$ & $K_{-1}^{\circ}$ & $K_{2}^{\circ}$ & $K_{-2}^{\circ}$ & $K_{\mathrm{c} 3}^{\circ}$ \\
\hline $3(\mathrm{~b})$ & $10^{-8}$ & $10^{-8}$ & $10^{-7}$ & $10^{-7}$ & $10^{-7}$ \\
$3(\mathrm{c})$ & $10^{-7}$ & $10^{-7}$ & $10^{-8}$ & $10^{-8}$ & $10^{-7}$ \\
$3(\mathrm{~d})$ & $10^{-7}$ & $10^{-7}$ & $10^{-7}$ & $10^{-7}$ & $10^{-8}$ \\
$3(\mathrm{e})$ & $10^{-8}$ & $10^{-8}$ & $10^{-8}$ & $10^{-8}$ & $10^{-8}$ \\
\hline
\end{tabular}


Table 3. Rate constants (in $\mathrm{mol} \mathrm{cm} \mathrm{cm}^{-2} \mathrm{~s}^{-1}$ ) used in the simulation of the ECE-mechanism.

\begin{tabular}{lccccc}
\hline Fig. & $K_{1}^{\circ}$ & $K_{-1}^{\circ}$ & $K_{\mathrm{c} 2}^{\circ}$ & $K_{-\mathrm{c} 2}^{\circ}$ & $K_{3}^{\circ}$ \\
\hline $4(\mathrm{~b})$ & $10^{-8}$ & $10^{-8}$ & $10^{-7}$ & $10^{-7}$ & $10^{-7}$ \\
$4(\mathrm{c})$ & $10^{-7}$ & $10^{-7}$ & $10^{-8}$ & $10^{-8}$ & $10^{-7}$ \\
$4(\mathrm{~d})$ & $10^{-7}$ & $10^{-7}$ & $10^{-7}$ & $10^{-7}$ & $10^{-8}$ \\
$4(\mathrm{e})$ & $10^{-8}$ & $10^{-8}$ & $10^{-7}$ & $10^{-7}$ & $10^{-8}$ \\
\hline
\end{tabular}

Table 4. Rate constants (in $\mathrm{mol} \mathrm{cm} \mathrm{cm}^{-2} \mathrm{~s}^{-1}$ ) used in the simulation of the parallel mechanism.

\begin{tabular}{lcccccc}
\hline Fig. & $K_{1}^{\circ}$ & $K_{-1}^{\circ}$ & $K_{2}^{\circ}$ & $K_{\mathrm{c3}}^{\circ}$ & $K_{-\mathrm{c} 3}^{\circ}$ & $K_{4}^{\circ}$ \\
\hline $5(\mathrm{~b})$ & $10^{-8}$ & $10^{-8}$ & $10^{-7}$ & $10^{-7}$ & $10^{-7}$ & $10^{-7}$ \\
$5(\mathrm{c})$ & $10^{-7}$ & $10^{-7}$ & $10^{-8}$ & $10^{-7}$ & $10^{-7}$ & $10^{-7}$ \\
$5(\mathrm{~d})$ & $10^{-7}$ & $10^{-7}$ & $10^{-7}$ & $10^{-7}$ & $10^{-7}$ & $10^{-8}$ \\
$5(\mathrm{e})$ & $10^{-7}$ & $10^{-7}$ & $10^{-7}$ & $10^{-8}$ & $10^{-8}$ & $10^{-7}$ \\
\hline
\end{tabular}

This reaction has been postulated for the dissolution of zinc. ${ }^{11,12}$ The monovalent $\mathrm{Zn}_{\mathrm{ad}}^{+}$is an intermediate which is adsorbed on the surface and is unstable in solution. The steady-state polarization curve, Fig. 1(a), shows Tafel slopes of 40 or $120 \mathrm{mV} /$ decade of current $\left(\alpha=\frac{1}{2}\right)$ depending on the rate-determining step. The impedance of this mechanism has been simulated with four different combinations of rate constants at five potentials. The impedance behaviour is shown in Figs. 1(b)-(e). The high-frequency loop is associated with the charge transfer of the reaction, while the second is the relaxation of the adsorbed intermediate. This second loop may be inductive or capacitive, depending on the choice of rate constants. For this mechanism it can be shown that if $K_{2}>K_{1}+K_{-1}(\partial I / \partial \theta>0)$ the relaxation of $\mathrm{Zn}_{\mathrm{ad}}^{+}$is inductive and vice versa. Thus, if the first step is in pseudo-equilibrium [Fig. 1(b)], two capacitive semicircles will appear. If, on the other hand, the second step is slightly faster, a very small inductive loop will show up [Fig. 1(c)]. However, this loop is very sensitive to anodic polarization, or to the choice of $K_{2}$ compared to $K_{1}$ and $K_{-1}$. For example, if $K_{2} /\left(K_{1}+K_{-1}\right) \geq 5$ and an anodic polarization of more than $30 \mathrm{mV}$ is simulated [Fig. 1(d)], the inductive loop will disappear and the Cole-Cole plot reveals only the charge-transfer loop. Fig. 1(e) shows the impedance spectra when all the rate constants are equal at $E=E^{\circ}$. At low potentials a small capacitive loop is present which disappears upon anodic polarization.

If the time constants are well separated, the diameter of the charge transfer loop gives the charge-transfer resistance directly. The diameter of the other semicircle depends on the rate ratio between the first and second electron-transfer steps. If the second step is very small compared to the first a large semicircle in the capacitive area of the $Z$-plane will be observed which becomes smaller as the rate of the second step is increased. If the second step is slightly faster than the first the diameter of the inductive semicircle goes through a maximum as the rate of the second electron transfer is made faster compared to the first. Thus if

Table 5. Parameters used in the simulations of the mechanisms.

\begin{tabular}{|c|c|c|c|c|c|}
\hline \multirow[t]{2}{*}{ Parameter } & \multicolumn{5}{|l|}{ Mechanism } \\
\hline & EE & EE-catalytic & EEC & ECE & Parallel E(E/CE) \\
\hline al/ä & $\begin{array}{l}F b\left[K_{1}+\left(K_{2}-K_{1}\right.\right. \\
\left.\left.+K_{-1}\right) \theta_{1, \mathrm{ss}}\right]\end{array}$ & $\begin{array}{l}F b\left[K_{1}+\left(4 K_{2}-K_{1}\right.\right. \\
\left.\left.+K_{-1}\right) \theta_{1, s s}\right]\end{array}$ & $\begin{array}{l}F b\left[K_{1}+\left(K_{2}-K_{1}+K_{-1}\right) \theta_{1, s s}\right. \\
\left.-\left(K_{1}-K_{-2}\right) \theta_{2, s s}\right]\end{array}$ & $\begin{array}{l}F b\left[K_{1}+\left(K_{-1}-K_{1}\right) \theta_{1, \mathrm{ss}}\right. \\
\left.+\left(K_{3}-K_{1}\right) \theta_{2, \mathrm{ss}}\right]\end{array}$ & $\begin{array}{l}F b\left[K_{1}+\left(K_{2}-K_{1}+K_{-1}\right) \theta_{1, \mathrm{ss}}\right. \\
\left.+\left(K_{3}-K_{1}\right) \theta_{2, \mathrm{ss}}\right]\end{array}$ \\
\hline$\partial / / \partial \theta_{1}$ & $F\left(K_{2}-K_{1}-K_{-1}\right)$ & $F\left(2 K_{2}-K_{1}-K_{-1}\right)$ & $F\left(K_{2}-K_{1}-K_{-1}\right)$ & $-F\left(K_{1}+K_{-1}\right)$ & $F\left(K_{2}-K_{1}-K_{-1}\right)$ \\
\hline$\partial / / \partial \theta_{2}$ & & & $-F\left(K_{1}+K_{-2}\right)$ & $F\left(K_{3}-K_{1}\right)$ & $F\left(K_{4}-K_{1}\right)$ \\
\hline & $K_{1}$ & $K_{1}$ & $K_{1}$ & $K_{1}$ & $K_{1}$ \\
\hline$\theta_{1, s s}$ & $\overline{K_{1}+K_{-1}+K_{2}}$ & $\overline{K_{1}+K_{-1}}$ & $\overline{K_{1}+K_{-1}+K_{2}\left(1+\frac{K_{1}-K_{-2}}{K_{\mathrm{c} 3}+K_{-2}}\right)}$ & $\overline{K_{1}+K_{-1}+K_{\mathrm{c} 2}\left(1+\frac{K_{1}-K_{-\mathrm{c} 2}}{K_{3}+K_{-\mathrm{c} 2}}\right)}$ & $K_{1}+K_{-1}+K_{2}+K_{\mathrm{c} 3}\left(1+\frac{K_{1}-K_{-\mathrm{c} 3}}{K_{4}+K_{-\mathrm{c} 3}}\right)$ \\
\hline$\theta_{2, \mathrm{ss}}$ & & & $\frac{K_{2} \theta_{1, \text { ss }}}{K_{3}+K_{-2}}$ & $\frac{K_{\mathrm{c} 2} \theta_{1, \mathrm{ss}}}{K_{3}+K_{-\mathrm{c} 2}}$ & $\frac{K_{\mathrm{c3}} \theta_{1, \mathrm{ss}}}{K_{4}+K_{-\mathrm{c} 3}}$ \\
\hline & $\beta$ & $\beta$ & $\beta$ & $\beta$ & $\beta$ \\
\hline$\tau_{\text {ads1 }}$ & $\overline{K_{1}+K_{-1}+K_{2}}$ & $\overline{K_{1}+K_{-1}}$ & $\overline{K_{1}+K_{-1}+K_{2}}$ & $\overline{K_{1}+K_{-1}+K_{2}}$ & $\overline{K_{1}+K_{-1}+K_{2}+K_{\mathrm{c} 3}}$ \\
\hline & & & $\beta$ & $\beta$ & $\beta$ \\
\hline$\tau_{\text {ads2 }}$ & & & $\overline{K_{3}+K_{-2}}$ & $\overline{K_{3}+K_{-\mathrm{c} 2}}$ & $\overline{K_{4}+K_{-c 3}}$ \\
\hline$I_{\mathrm{ss}}$ & $2 K_{2} \theta_{1, \mathrm{ss}}$ & $2 K_{2} \theta_{1, \text { ss }}$ & $2\left(K_{2} \theta_{1, \mathrm{ss}}-K_{-2} \theta_{2, \mathrm{ss}}\right)$ & $2 K_{2} \theta_{1, \mathrm{ss}}+\left(K_{3}-K_{-\mathrm{c} 2}\right) \theta_{2, \mathrm{ss}}$ & $\left(2 K_{2}+K_{\mathrm{c} 3}\right) \theta_{1, \mathrm{ss}}+\left(K_{4}-K_{-\mathrm{cs}}\right) \theta_{2, \mathrm{ss}}$ \\
\hline
\end{tabular}




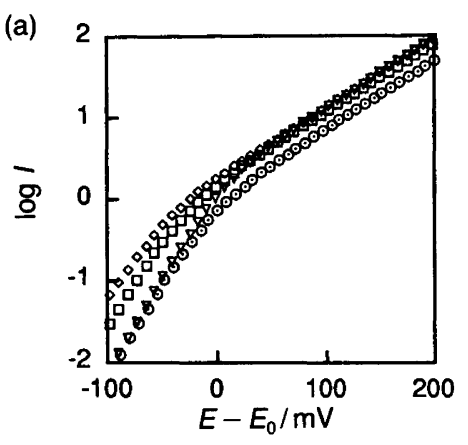

(c)

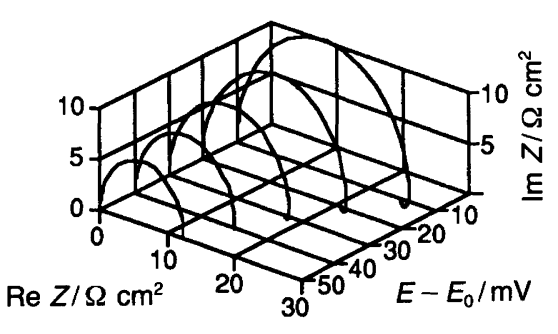

(e)

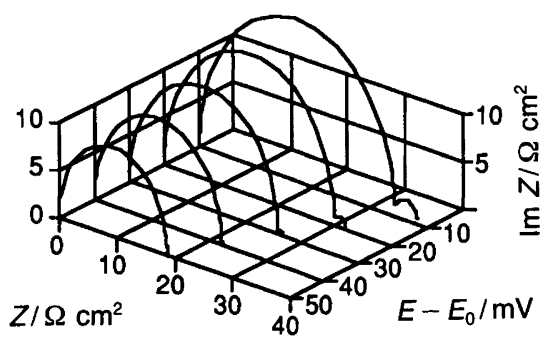

(b)

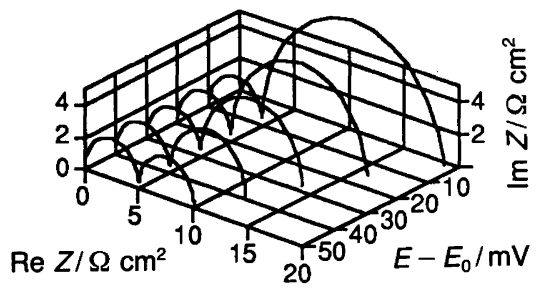

(d)

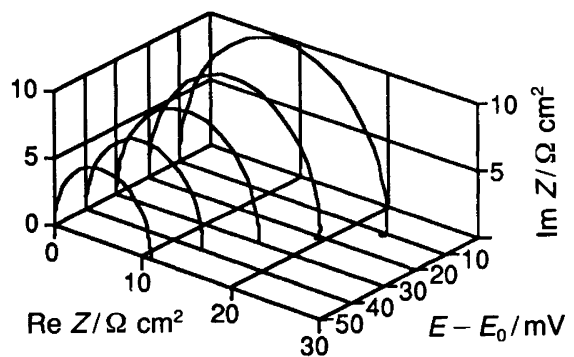

Fig. 1. Simulated polarization curves (a) and impedance spectra [(b)-(e)] for the EEmechanism. (Impedance spectra simulated at $10,20,30$, 40 and $50 \mathrm{mV}$.) (a) $\diamond$. First step r.d.s.; $\nabla$, second step r.d.s.; $\square$, second step slightly faster than the first; $\odot$, all rate constants equal at $E^{\circ}$.

(b) Second step r.d.s. at $E^{\circ}$. (c) Second step slightly faster than the first at $E^{\circ}$. (d) First step r.d.s. at $E^{\circ}$. (e) All rate constants equal at $E^{\circ}$.
$K_{2} \gg\left(K_{1}+K_{-1}\right)$ no relaxation from the adsorbed species will be seen.

\section{Catalytic EE-mechanism:}

$\mathrm{Zn} \underset{k_{-1}}{\stackrel{k_{1}}{\rightleftharpoons}} \mathrm{Zn}_{\theta_{1}^{+}} \underset{-2 \mathrm{e}^{-}}{\stackrel{k_{2}}{\longrightarrow}} \mathrm{Zn}_{\mathrm{aq}}^{2+}+\underset{\theta_{1}}{\mathrm{Zn}_{\mathrm{ad}}^{+}}$

This reaction sequence is similar to the one proposed by Heusler ${ }^{13}$ for the dissolution of iron. The mechanism has also been suggested by some groups ${ }^{14-16}$ for zinc dissolution in chloride and sulfate media. The reaction of the $\mathrm{Zn}_{\mathrm{ad}}^{+}$ intermediate formed in the first step is catalysed by the presence of kink sites in the zink lattice. The $\mathrm{Zn}$ atom in this kink site, $\mathrm{Zn}_{\mathrm{k}}$, is simultaneously oxidised to $\mathrm{Zn}_{\mathrm{ad}}^{+}$. Thus, the number of electrons transfered in the autocatalytic step is two. As a result, the Tafel slopes from the steady-state polarization curve [Fig. 2(a)] are changed from
40 and $120 \mathrm{mV}$ (the non-catalytic EE-mechanism) to 30 and $60 \mathrm{mV}$, respectively. The mechanism was simulated under the same conditions as the previous EE-mechanism, and its impedance behaviour is shown in Figs. 2(b)-(e). The conditions for inductive and capacitive behaviour in the lowfrequency part of the spectrum are the same as for the EE-mechanism. That is, when the first step is in pseudoequilibrium [Fig. 2(b)], the response of the $\mathrm{Zn}_{\mathrm{ad}}^{+}$species will be capacitive and vice versa [Figs. 2(c) and (d)]. However, the diameter of the inductive loop in the catalytic mechanism is larger and remains in a broader range of anodic potentials. The stability of the inductive loop towards anodic polarization is due to a different potential dependence of the time constant compared to its non-catalytic equivalent. Fig. 2(e) shows the impedance spectra when all the rate constants are equal at $E=E^{\circ}$. In contrast to the EE-mechanism, the first step becomes the rate-determining step at very low overpotentials, which results in an inductive loop in the low-frequency region that also disappears upon anodic polarization. 


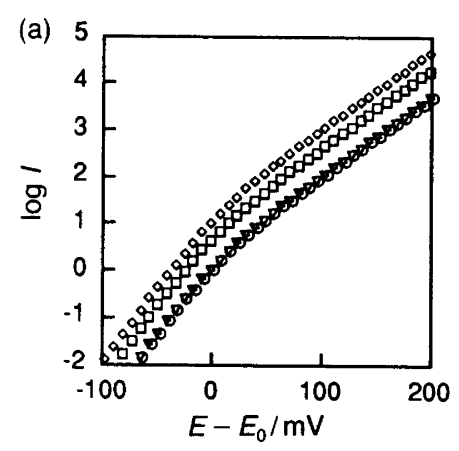

(c)

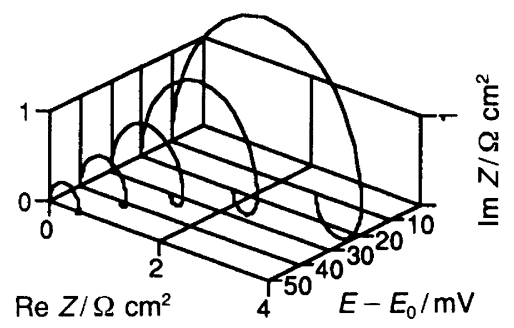

(e)

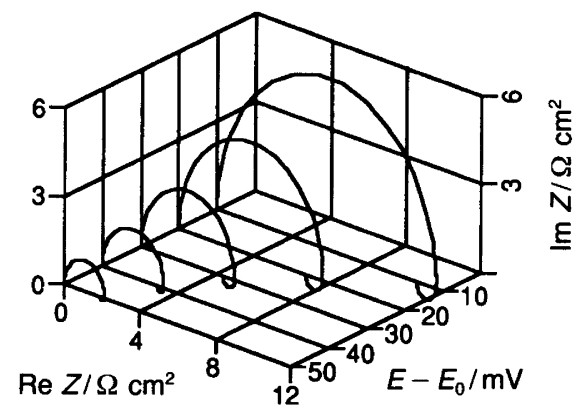

EEC-mechanism:

$\mathrm{Zn} \underset{k_{-1}}{\stackrel{k_{1}}{\rightleftharpoons}} \mathrm{Zn}_{\theta_{1}^{+}}^{\stackrel{k_{2}}{\rightleftharpoons}} \underset{k_{-2}}{\rightleftharpoons} \mathrm{Zn}_{2}^{2+} \stackrel{k_{\mathrm{cd}}}{\longrightarrow} \mathrm{Zn}_{\mathrm{aq}}^{2+}$

This reaction sequence has been suggested by Cachet and Wiart ${ }^{14,15}$ as an alternative and parallel dissolution path to the catalytic mechanism. The polarization behaviour is shown in Fig. 3(a). It can be seen that if the chemical step is fast compared to the electrochemical steps this mechanism shows the same steady-state behaviour as the EE-mechanism (40 and $120 \mathrm{mV}$ Tafel slopes). If, on the other hand, the chemical step is the rate-determining step, the steadystate curve shows a $30 \mathrm{mV}$ Tafel slope that ends in a potential-independent region at anodic potentials. The impedance behaviour is shown in Figs. 3(b)-(e).
This mechanism shows an impedance behaviour that differs in one respect from the other mechanisms: the polarization resistance measured as the difference between the impedance at zero and infinite frequency becomes negative under certain conditions. This behaviour is often seen when metals are passivated by an oxide film. ${ }^{17}$ The polarization curves of these metals usually show negative slopes, which explains the negative polarization resistance. Thus, the result of the impedance simulation of this mechanism is somewhat unexpected, since the current slope, although almost independent of the potential, is still positive. A possible explanation of this behaviour might be that there is interference between the two relaxations of the intermediates. For example, it is noted for this mechanism that the sign of the steady-state value of $\Delta \theta_{\mathrm{Zn}_{a d}^{+}} / \Delta E$ (the real part of the complex quantity) is the same as the sign of the polarization resistance. We have for comparison simulated the 
(a)

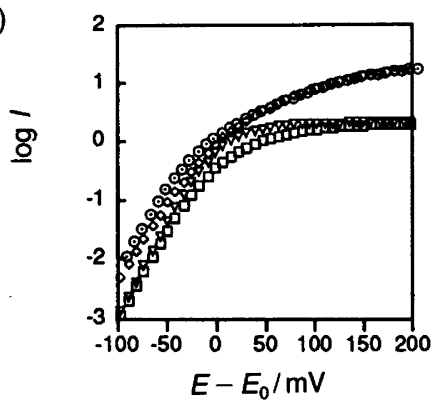

(c)

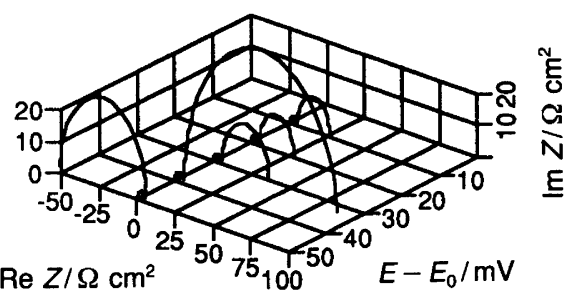

(e)

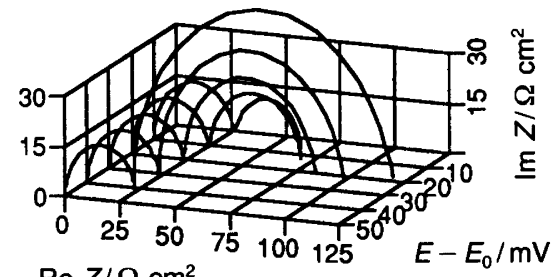

$\operatorname{Re} Z / \Omega \mathrm{cm}^{2}$
$\operatorname{Re} Z / \Omega \mathrm{cm}^{2}$ (b)

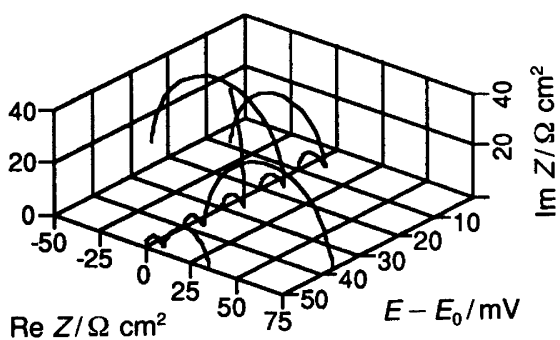

(d)

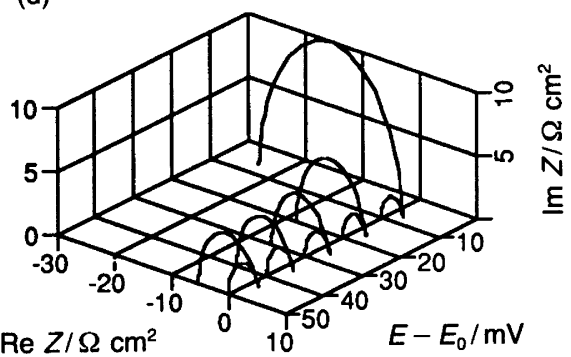

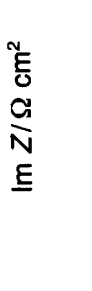

$\operatorname{Re} Z / \Omega \mathrm{cm}^{2}$

$$
E-E_{0} / \mathrm{mV}
$$

Fig. 3. Simulated polarization curves (a) and impedance spectra [(b)-(e)] for the EECmechanism. (Impedance spectra simulated at $10,20,30$, 40 and $50 \mathrm{mV}$.) (a) $\odot$, First step r.d.s.; $\diamond$, second step r.d.s.; $\nabla$, third step r.d.s.; $\square$, all rate constants equal at $E^{\circ}$.

(b) First step r.d.s. at $E^{\circ}$.

(c) Second step r.d.s. at $E^{\circ}$.

(d) Third step r.d.s. at $E^{\circ}$. (e) All rate constants equal at $E^{\circ}$. 


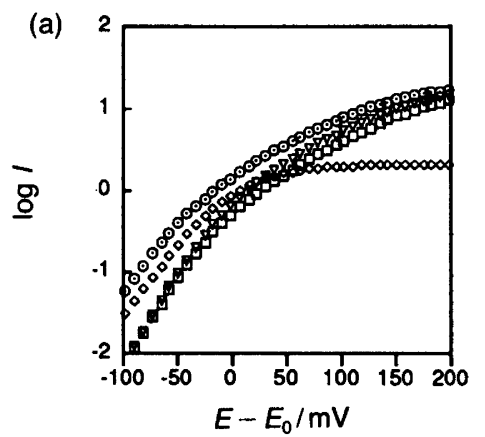

(c)

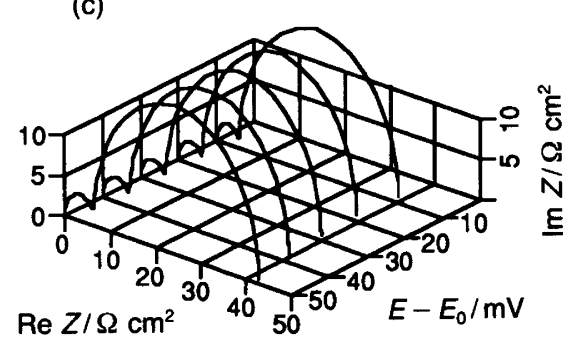

(e)

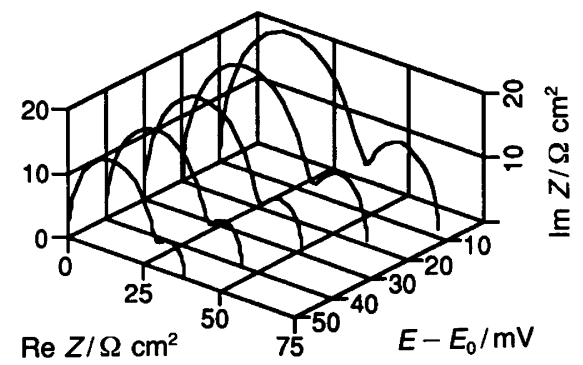

since the relaxation times of the intermediates are of the same order. As the potential is increased the diameter is decreased. If the chemical step is slow [Fig. 4(c)], two capacitive loops are also observed. The second loop in the low-frequency part of the spectra is, however, increased upon anodic polarization, while the charge-transfer loop remains fairly independent of the potential. Fig. 4(d) shows the spectra when the second electron transfer is rate-determining. One of the relaxations of the intermediates becomes inductive in this case and three separate loops are resolved. We noted that this inductive loop showed up whenever $\partial I / \partial \theta_{\mathrm{Zn}}{ }^{*}\left[=F\left(K_{3}-K_{1}\right)\right]$ for this mechanism was negative. Fig. 4(e) shows the spectra when the chemical step is in equilibrium. The fast chemical step makes the relaxation times of the adsorbed intermediates comparable with the relaxation of the charge-transfer process. It is therefore notable that two loops appear in the spectra. This is discussed in the next section. Negative polarization resistance was never observed in the simulation of this mechanism.
Parallel E(E/CE)-mechanism:

$$
\begin{aligned}
& \mathrm{Zn} \underset{k_{-1}}{\stackrel{k_{1}}{\rightleftharpoons}} \underset{\theta_{1}}{\mathrm{Zn}_{\mathrm{ad}}^{+}} \stackrel{k_{2}}{\longrightarrow} \mathrm{Zn}_{\mathrm{aq}}^{2+} \\
& k_{\mathrm{c} 3} \| k_{-\mathrm{c} 3}
\end{aligned}
$$

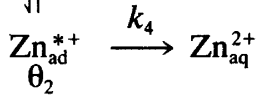

This mechanism consists of two dissolution routes. One route is the same as the EE-mechanism and the other is the same as the ECE-mechanism. Both mechanisms have been discussed earlier, separately. The first electron transfer and the $\mathrm{Zn}_{\mathrm{ad}}^{+}$species are common for both paths. Fig. 5(a) shows the polarization curves for the mechanism when different steps are rate-determining. They are all rather similar, with 40/120 mV Tafel slopes. When the second step in the EE-path is rate-determining at $E^{\circ}$ there is a limited potential region where the chemical step becomes impor- 


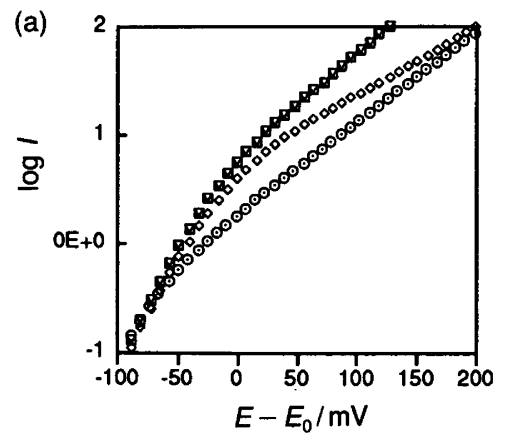

(c)

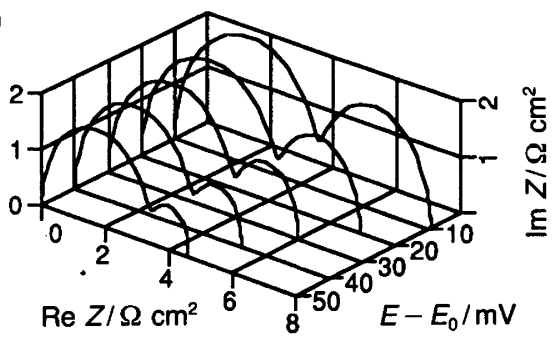

(e)

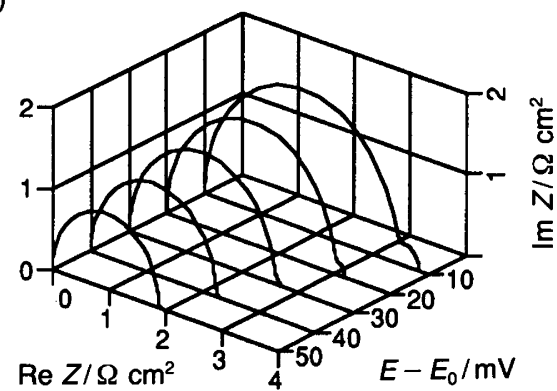

(b)

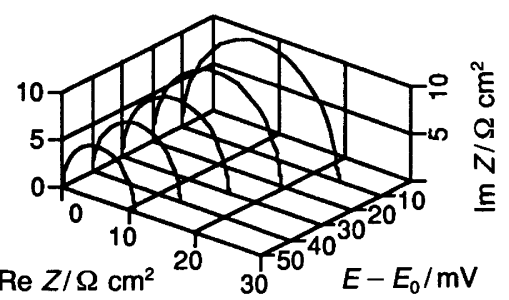

(d)

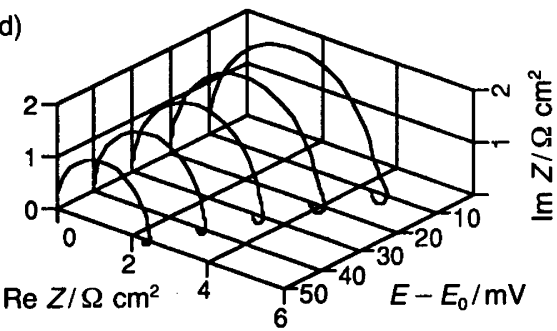

Fig. 5. Simulated polarization curves (a) and impedance spectra [(b)-(e)] for the $E(E / C E)$-mechanism. (Impedance spectra simulated at $10,20,30,40$ and $50 \mathrm{mV}$.) (a) $\odot$, First step r.d.s.; $\diamond$, second step r.d.s. (K$K_{2}$-step); $\nabla$, fourth step r.d.s. $\left(K_{4}\right.$-step); $\square$, chemical step r.d.s. at $E^{\circ}$.

(b) First step r.d.s. at $E^{\circ}$.

(c) Second step r.d.s. ( $K_{2}$-step) at $E^{\circ}$. (d) Fourth step ( $K_{4}$-step) r.d.s. at $E^{\circ}$. (e) Chemical step r.d.s. at $E^{\circ}$.

\section{ह N}


(a)

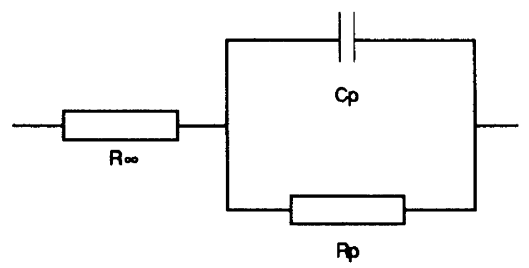

(b)

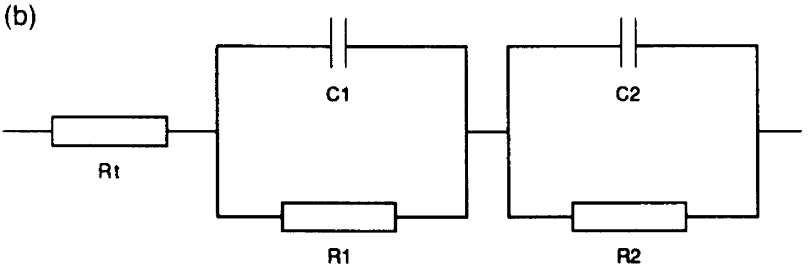

Fig. 6. (a) Equivalent circuit originally suggested by Gerischer to describe a charge-transfer process with one adsorbed intermediate. (b) Equivalent circuit for a charge-transfer process with two adsorbed intermediates.

corresponding analytical solution of the model in the impedance domain. The choice of the circuit is not crucial as long as its impedance can be expressed in the same form as one of the analytical solutions. The equivalent circuit used with this approach should therefore not be regarded as a model of the electrochemical system but merely as a mathematical tool to obtain information about parameters describing the system.

An example of this information transfer is the evaluation of the time constants associated with the adsorbed intermediates from the experimental spectrum. Armstrong has discussed this problem for reactions with one adsorbed intermediate.$^{19}$ The faradaic admittance can be written as eqn. (26), where $R_{\infty}$ is the infinite-frequency charge-

$Y_{\mathrm{f}}=\frac{1}{R_{\infty}}+\frac{1}{R_{0}} \frac{1}{1+\mathrm{i} \omega \tau}$

transfer resistance and $R_{0}$ an additional resistance at zero frequency. This expression is of the same form as the analytical solution for the faradaic admittance presented earlier in this paper [eqn. (21), $R_{0}=T / B$ and $\tau=1 / T$ ]. If the equivalent circuit suggested in early works of Gerischer $^{20,21}$ [Fig. 6(a)] is used, the time constant may be obtained from eqn. (27), where $\omega^{*}$ is the frequency

$\tau=\frac{1}{\omega^{*}} \frac{R_{0}+R_{\infty}}{R_{0}}$

obtained at the maximum of the second semicircle. $R_{0}$ and $R_{\infty}$ are related to the parallel resistance $R_{\mathrm{p}}$ and capacitance $C_{\mathrm{p}}$ in the circuit in Fig. 6(a) by eqn. (28).

$R_{\mathrm{p}}=\frac{-R_{\infty}^{2}}{R_{0}+R_{\infty}} \quad C_{\mathrm{p}}=\frac{-R_{0} \tau}{R_{\infty}^{2}}$
The situation becomes more complex when this treatment is generalized to included additional relaxations. The analytical solutions of the faradaic admittance for two or three relaxations [eqns. (22) and (23)] show that the number of unknown parameters $J_{i j}$ increases by a power of two to the number of relaxations. Thus, it is not possible without any assumptions to obtains information concerning the individual time constants associated with the adsorbed intermediates. However, since the parameter $T$ is the sum of the inverse of time constants involved in the faradaic process, it is possible to estimate the slowest time constant if it is well separated from the other constants. Suppose we want to estimate the relaxation times for a chemical reaction with two intermediates from a spectrum. The impedance of the circuit in Fig. 6(b) can be written as eqn. (29),

$\mathrm{Z}_{\mathrm{c}}=R_{\mathrm{t}}+\frac{\left[\left(R_{1}+R_{2}\right)\right] / \tau_{1} \tau_{2}+\mathrm{i} \omega\left[\left(R_{1} \tau_{2}+R_{2} \tau_{1}\right) / \tau_{1} \tau_{2}\right]}{1 / \tau_{1} \tau_{2}-\omega^{2}+\mathrm{i} \omega\left[\left(\tau_{1}+\tau_{2}\right) / \tau_{1} \tau_{2}\right]}$

where $\tau_{1}$ and $\tau_{2}$ are $R_{1} C_{1}$ and $R_{2} C_{2}$, respectively. If these values can be estimated from the experimental spectrum we can transform eqn. (22) into the impedance plane, eqn. (30). Eqns. (31)-(34) can then be set.

$Z_{\mathrm{f}}=R_{\mathrm{t}}+\frac{-R_{\mathrm{t}}^{2} A-\mathrm{i} \omega \beta R_{\mathrm{t}}^{2} B}{D+R_{\mathrm{t}} A-\omega^{2} \beta^{2}+\mathrm{i} \omega \beta\left(T+R_{\mathrm{t}} B\right)}$

$A=-\frac{1}{R_{\mathrm{t}}^{2} C_{1} C_{2}}\left(\frac{1}{R_{1}}+\frac{1}{R_{2}}\right)$

$B=-\frac{1}{R_{\mathrm{t}}^{2}}\left(\frac{1}{C_{1}}+\frac{1}{C_{2}}\right)$

$D=\frac{1}{\tau_{1} \tau_{2}}-R_{\mathrm{r}} A$

$T=\left(\frac{1}{\tau_{1}}+\frac{1}{\tau_{2}}\right)-R_{\mathrm{t}} B$

These expressions may now be identified with the corresponding parameters derived from the particular mechanism of interest. If we look at the relaxation times of the adsorbed intermediates we note that these are related to $T$ by eqn. (35), where $\tau_{\mathrm{ads} 1}=-1 / J_{11}$ and $\tau_{\mathrm{ads} 2}=-1 / J_{22}$. If

$T=1 / \tau_{\text {ads } 1}+1 / \tau_{\text {ads } 2}$

$\tau_{\text {ads1 }} \gg \tau_{\text {ads2 }}$ then $T \approx 1 / \tau_{\text {ads2 }}$, where $\tau_{\text {ads1 }}$ and $\tau_{\text {ads2 }}$ are the time constants associated with the adsorbed intermediates. The time constant $\tau_{\text {ads } 2}$ is then from eqn. (34), given by eqn. (36).

$\tau_{\mathrm{ads} 2}=\frac{1}{\left(\frac{1}{\tau_{1}}+\frac{1}{\tau_{2}}\right)-R_{\mathrm{t}} B}$ 
The weak point here is of course the justification of the assumption that the time constants $\tau_{\text {ads } 1}$ and $\tau_{\text {ads } 2}$ are of different magnitude. We have in fact no information about these time constants and their relative magnitudes. Nevertheless, we think the derivation is motivated, because it shows that the relationship between the observed experimental time constants and the time constants of the adsorbed intermediates, as they are defined from the derivation of the impedance, is not straightforward. For example, the following fact was noticed when the ECEmechanism was simulated with the chemical step in equilibrium. The time constants of the two intermediates, derived from the calculation of the impedance, were approximately the same as the time constant of the charge-transfer process coupled with the double-layer capacitance. Consequently, we expected one loop in the impedance plane diagram. Two capacitive loops were, however, observed. Analysis of the spectra in terms of the equivalent circuit shown in Fig. 6(b) gave the following values of the components involved: $R_{1}=0.33 \Omega, \tau_{1}=4.95 \times 10^{-3} \mathrm{~s}\left(=>C_{1}=15 \mathrm{mF}\right), R_{2}=22.2 \Omega$, $\tau_{2}=2.99 \times 10^{-2} \mathrm{~s}\left(=>C_{2}=1.35 \mathrm{mF}\right)$ and $R_{\mathrm{t}}=47.1 \Omega$, $\tau_{\mathrm{t}}=7.06 \times 10^{-3} \mathrm{~s}$. The time constants $\tau_{1}$ and $\tau_{2}$ should be compared with the time constants $\tau_{\text {ads } 1}$ and $\tau_{\text {ads } 2}$ associated with the intermediates. These were calculated from the derivation of the impedance and are $\tau_{\mathrm{ads} 1}=\tau_{\mathrm{ads} 2}=$ $9.80 \times 10^{-3} \mathrm{~s}$.

The use of the charge-transfer resistance or polarization resistance in corrosion rate measurements with IS was discussed in the literature some years ago. ${ }^{22,23}$ The French school, with Keddam et al., argued for the use of $R_{\mathrm{t}}$ in order to obtain the true corrosion rate, while Lorenz $e t$ al. stated that $R_{\mathrm{p}}$ should be used instead. The problem was, as far as we can see, an empirical one, since other methods such as weight loss determinations and solution analysis did not agree with the impedance data. Although the main purpose of this paper is not the determination of corrosion rates, we became interested in how $R_{\mathrm{t}}$ and $R_{\mathrm{p}}$ were related to the steady-state current in order to investigate the possibility of estimating Tafel slopes from these quantities. In principal this should not be a problem.

The inverse of the charge-transfer resistance at steady state, $1 / R_{\mathrm{t}}$, is defined by eqn. (37), where all other state

$\frac{1}{R_{\mathrm{t}}}=\frac{\mathrm{d} I_{\mathrm{ss}}}{\mathrm{d} E}$

variables depending on the potential, $E$, are kept fixed. The inverse of the polarization resistance, $1 / R_{\mathrm{p}}$, is defined by eqn. (38), where $i$ is the number of state variables,

$\frac{1}{R_{\mathrm{p}}}=\frac{1}{R_{\mathrm{t}}}+\sum_{i} \frac{\partial I_{\text {iss }}}{\partial X_{i s \mathrm{~s}}} \frac{\partial X_{\text {iss }}}{\partial E}$

$X$, that depend on the potential. With these definitions a simple and inverse relationship between $R_{\mathrm{p}}$ and $I_{\mathrm{ss}}$ for all potentials can be derived. The Tafel slope could then be estimated by $\ln (10) R_{\mathrm{p}} I$ or the variation of $-\log R_{\mathrm{p}}$ with the potential. The $R_{\mathrm{t}}$ value can only be used if the potential is such that no reaction step in the mechanism is in equilibrium with its adjacent step or steps. We have, for simple and pure electrochemical mechanisms such as the concecutive EE-mechanisms (1) and (2), which are often used in metal dissolution reactions, investigated the potential dependence of $R_{\mathrm{p}}$ and $R_{\mathrm{t}}$, and the statements above hold as long as the mechanisms consist of electrochemical steps. If potential-independent steps are introduced into the mechanism the simple relationship between $R_{\mathrm{p}}$ and $I_{\mathrm{ss}}$ disappears and the Tafel slope has to be estimated through $R_{\mathrm{t}}$, which in turn can only be used at potentials far from equilibrium.

\section{Conclusions}

(1) A combination of polarization and impedance measurements constitutes a powerful tool in mechanistic analysis. (2) With a mechanism involving two or more intermediates it is not possible to obtain an unambiguous relationship between the experimental time constants and the time constants obtained from the model. (3) There is a simple and inverse relationship between $R_{\mathrm{p}}$ and the steady-state current if the mechanism consists of electrochemical steps only. This simple relationship is lost when chemical steps are introduced into the mechanism, which in turn makes it impossible to use the value of $\ln (10) R_{\mathrm{p}} I_{\mathrm{ss}}$ as a true Tafel value.

Acknowledgements. This research project was financially supported by AB Volvo, Göteborg, Sweden. The authors thank Dr. G. Wirmark and Dr. G. Ström for fruitful discussions.

\section{Appendix I}

List of symbols used.

$\alpha \quad$ transfer coefficient

b $\quad \alpha z F / R T$

$\beta_{i} \quad$ maximum surface concentration of species $i$

$C_{\mathrm{dl}} \quad$ double layer capacitance

$E$ potential

$E^{\circ} \quad$ equilibrium potential

$E_{\mathrm{ss}} \quad$ steady-state potential

$F \quad$ Faraday constant

$\varphi \quad$ phase angle

$f \quad$ frequency

$i \quad$ index of adsorbed species

I current density

$I_{\mathrm{f}} \quad$ faradaic current density

$I_{\text {ss }} \quad$ steady-state current density

i complex number, $V-1$

$K_{n}^{\circ} \quad$ normalized rate constant at equilibrium potential

$k_{n}^{\circ} \quad$ rate constant at equilibrium potential

$K_{\text {c } n} \quad$ normalized chemical rate constant 
$K_{n} \quad$ normalized rate constant

$k_{n} \quad$ rate constant

$m$ number of adsorbed species in a certain mechanism

$v \quad$ normalized rate of adsorption

$\theta_{i} \quad$ fraction coverage by species $i$

$\theta_{i, \mathrm{ss}} \quad$ fraction coverage by species $i$ at steady state

$R_{\mathrm{t}} \quad$ charge transfer resistance

$t$ time

$\tau_{i} \quad$ time constant of the relaxation by species $i$

$\omega$ angular frequency, $2 \pi f$

$Y_{\mathrm{f}}$ faradaic admittance

$z \quad$ number of electrons involved in a reaction step

$Z_{\mathrm{f}} \quad$ faradaic impedance

$Z_{\mathrm{I}} \quad$ interfacial impedance

Parameters used in the impedance calculations, common for all mechanisms.

$$
\begin{aligned}
& A=m_{1} a_{2} J_{12}+m_{2} a_{1} J_{21}-m_{1} a_{1} J_{22}-m_{2} a_{2} J_{11} \\
& A^{\prime}=-m_{1} a_{1} L_{11}+m_{1} a_{2} L_{21}-m_{1} a_{3} L_{31} \\
& +m_{2} a_{1} L_{12}-m_{2} a_{2} L_{22}+m_{2} a_{3} L_{32} \\
& -m_{3} a_{1} L_{13}+m_{3} a_{2} L_{23}-m_{3} a_{3} L_{33}
\end{aligned}
$$

$a_{i}=\frac{\partial\left(\frac{\mathrm{d} \theta_{i}}{\mathrm{~d} t}\right)}{\partial E} ;$ the right-hand side of the linear equation

$$
B=\sum_{i=1}^{m} m_{i} a_{i}
$$

$C=m_{1}\left[b_{1}\left(J_{22}+J_{33}\right)-a_{2} J_{12}-a_{3} J_{13}\right]$

$+m_{2}\left[-a_{1} J_{21}+a_{2}\left(J_{11}+J_{33}\right)-a_{3} J_{23}\right]$

$+m_{3}\left[-a_{1} J_{31}-a_{2} J_{32}+a_{3}\left(J_{11}+J_{22}\right)\right]$

$D=$ the determinant of the matrix $J$

$J_{i j}=\frac{\partial\left(\frac{\mathrm{d} \theta_{i}}{\mathrm{~d} t}\right)}{\partial \theta_{j}} \begin{aligned} & \text { these coefficients give the coefficient ma- } \\ & \begin{array}{l}\text { trix, } J, \text { that determines the linear equation } \\ \text { system }\end{array}\end{aligned}$

$L_{i j}=$ the subdeterminant of the matrix that is left when the $i$ th row and $j$ th column of $J$ are deleted

$$
m_{i}=\frac{\partial I}{\partial \theta_{i}}
$$

$S=\sum_{i=1}^{m} L_{i i}$

$$
-T=\sum_{i=1}^{m} \frac{\partial\left(\frac{\mathrm{d} \theta_{i}}{\mathrm{~d} t}\right)}{\partial \theta_{i}}
$$

\section{Appendix II}

Derivation of the impedance. The inverse of the faradaic impedance, $Z_{\mathrm{f}}$, is defined as $1 / Z_{\mathrm{f}}=\mathrm{d} I / \mathrm{d} E$ when the electrode is pertubated by a signal around the steady-state potential. If the signal is a sine-wave potential $\delta E$ with a small amplitude $|\delta E|$ it can be expressed as $\delta E=|\delta E|$ $\exp (i \omega t)$, where $\omega$ is the angular frequency $\left(\mathrm{s}^{-1}\right)$ and $\mathrm{i}=\mathrm{V}-1$. The signal causes the current, $I$, and all other state variables depending on $E$, to vary with the same frequency around their steady-state values. The faradaic impedance can be calculated using the Taylor expansion limited to the first order of the expression that describes the current flowing through the electrode. The derivation is made under assumptions that are commonly used for metal dissolution reactions and can be summarized as follows: (1) kinetically controlled reaction steps which follow the Tafel law, and (2) use of the Langmuir adsorption isotherm for the adsorption-desorption of the intermediates.

Consider the following mechanism:

$$
\begin{aligned}
& \mathrm{Zn} \underset{k_{-1}}{\stackrel{k_{1}}{\rightleftharpoons}} \underset{\theta_{1}}{\mathrm{Zn}_{\mathrm{ad}}^{+}} \stackrel{k_{2} \text {, cat. }}{\underset{\mathrm{Zn}_{\mathrm{k}}}{\longrightarrow}} \underset{\boldsymbol{\theta}_{1}}{\mathrm{Zn}_{\mathrm{ad}}^{+}}+\mathrm{Zn}_{\mathrm{aq}}^{2+}+2 \mathrm{e}^{-} \\
& k_{\mathrm{c} 4} \| k_{-\mathrm{c} 4} \\
& \underset{\boldsymbol{\theta}_{2}}{\mathrm{Zn}_{\mathrm{ad}}^{*+}} \stackrel{k_{3}}{\longrightarrow} \mathrm{Zn}_{\mathrm{aq}}^{2+}
\end{aligned}
$$

The rate of the individual reaction steps, $K_{n}$, is the product of the heterogeneous rate constant, $k_{n}$, and the concentration of the reacting species. The rate constant, $k_{n}$, is related to the potential by $k_{n}=k_{n}^{\circ} \exp \left(b_{n} E\right)$, where $k_{n}^{\circ}$ is the rate constant at $E^{\circ}$ and $b_{n}$ the Tafel coefficient. If the $K_{1}$ reaction only takes place on bare surface sites, the charge- and mass-balance are given by eqn. (A1) and eqns. (A2) and (A3), respectively, where $F$ is the Faraday constant. The

$I_{\mathrm{f}}=F\left[K_{1}\left(1-\theta_{1}-\theta_{2}\right)+2 K_{2} \theta_{1}+K_{3} \theta_{2}-K_{-1} \theta_{1}\right]$

$\beta_{1} \frac{\mathrm{d} \theta_{1}}{\mathrm{~d} t}=K_{1}\left(1-\theta_{1}-\theta_{2}\right)+K_{-\mathrm{c} 4} \theta_{2}-\left(K_{-1}+K_{\mathrm{c} 4}\right) \theta_{1}$

$\beta_{2} \frac{\mathrm{d} \theta_{2}}{\mathrm{~d} t}=K_{\mathrm{c} 4} \theta_{1}-\left(K_{-\mathrm{c} 4}+K_{3}\right) \theta_{2}$

steady-state solutions, $\theta_{i, \mathrm{ss}}$, of $\mathrm{d} \theta_{i} / \mathrm{d} t=0$, are given by eqns. (A4) and (A5). Substitution of $\theta_{i}$ by the corresponding 
$\theta_{1, \mathrm{ss}}=\frac{K_{1}}{K_{1}+K_{-1}+K_{\mathrm{c} 4}\left(1+\frac{K_{1}-K_{-\mathrm{c} 4}}{K_{-\mathrm{c} 4}+K_{3}}\right)}$

$\theta_{2, \mathrm{ss}}=\frac{K_{\mathrm{c} 4} \theta_{1}}{K_{-\mathrm{c} 4}+K_{3}}$

steady-state value into eqn. (A1) yields the steady-state current, which can be written as eqn. (A6). This equation

$I_{\mathrm{ss}}=F\left[\left(2 K_{2}+K_{\mathrm{c} 4}\right) \theta_{1}+\left(K_{3}-K_{-\mathrm{c} 4}\right) \theta_{2}\right]$

is used for the simulation of the steady-state current. The first-order Taylor expansion of eqn. (A1) divided by $\Delta E$ gives the inverse of the faradaic impedance, eqn. (A7),

$\frac{1}{Z_{\mathrm{f}}}=\left(\frac{\partial I}{\partial E}\right)_{\theta_{i}}+\sum_{i=1}^{2}\left(\frac{\partial I_{\mathrm{f}}}{\partial \theta_{i}}\right)_{E} \frac{\Delta \theta_{i}}{\Delta E}$

where the first term is the inverse of the charge-transfer resistance. If the transfer coefficients are the same for all steps, the charge-transfer resistance can be written as eqn. (A8). $\left(\partial I_{f} / \partial \theta_{i}\right)_{E}$ are easily obtained from eqn. (A1) as eqns. (A9) and (A10). The last factor in eqn. (A7),

$\frac{1}{R_{\mathrm{t}}}=F b\left[K_{1}\left(1-\theta_{1}-\theta_{2}\right)+4 K_{2} \theta_{1}+K_{-1} \theta_{1}+K_{3} \theta_{2}\right]$

$\frac{\partial I_{\mathrm{f}}}{\partial \theta_{1}}=F\left(2 K_{2}-K_{1}-K_{-1}\right)$

$\frac{\partial I_{\mathrm{f}}}{\partial \theta_{2}}=F\left(K_{3}-K_{1}\right)$

$\Delta \theta_{i} / \Delta E$, is obtained from eqns. (A2) and (A3) utilizing the result that, for a sine-wave variation of $\theta_{i}$, eqn. (A11) holds.

$\Delta \frac{\mathrm{d} \theta_{i}}{\mathrm{~d} t}=\frac{\mathrm{d}}{\mathrm{d} t} \Delta \theta_{i}=\mathrm{i} \omega \Delta \theta_{i}$

Thus the set of linear equations (A12) and (A13) can be

$\left(A_{1}+\mathrm{i} \omega \beta_{1}\right) \frac{\Delta \theta_{1}}{\Delta E}+B_{1} \frac{\Delta \theta_{2}}{\Delta E}=C_{1}$

$A_{2} \frac{\Delta \theta_{1}}{\Delta E}+\left(B_{2}+\mathrm{i} \omega \beta_{2}\right) \frac{\Delta \theta_{2}}{\Delta E}=C_{2}$

set up, in which the coefficients are given by eqns. (A14)-(A19).

$A_{1}=K_{1}+K_{-1}+K_{\mathrm{c} 4}$
$B_{1}=K_{1}-K_{-c 4}$

$C_{1}=b\left[K_{1}-\left(K_{1}-K_{-1}\right) \theta_{1}-K_{1} \theta_{2}\right]$

$A_{2}=-K_{\mathrm{c} 4}$

$B_{2}=K_{3}+K_{-c 4}$

$C_{2}=-b K_{3} \theta_{2}$

The time constants associated with the relaxation of the adsorbed intermediates are obtained from these equations by the relationships $\tau_{1}=\beta_{1} / A_{1}$ and $\tau_{2}=\beta_{2} / B_{2}$.

Solving the set of equations with respect to $\Delta \theta_{i} / \Delta E$ and substituting the result into eqn. (A7) yields the faradaic admittance, $1 / Z_{f}$, which after addition of the double-layer capacitance, $C_{\mathrm{dl}}$, in parallel to $Z_{\mathrm{f}}$ gives the interfacial impedance, $Z_{1}$, via eqn. (A20) or (A21).

$\frac{1}{Z_{\mathrm{I}}}=\frac{1}{Z_{\mathrm{f}}}+\mathrm{i} \omega C_{\mathrm{dl}}$

$Z_{\mathrm{I}}=\frac{Z_{\mathrm{f}}}{1+\mathrm{i} \omega Z_{\mathrm{f}} C_{\mathrm{dl}}}$

In order to obtain $\Delta \theta_{i} / \Delta E$, using the analytical solutions presented earlier, it is preferable to use a computer algorithm which can handle determinants. We have used the adjoint method for the solution of the linear equation system. The parameters $D, J$ and $L$ are determinants and subdeterminants of the coefficient matrix given by the linear equation system. When the first set of parameters are calculated these can be substituted into the next set, giving after the addition of the double-layer capacitance, the total electrode admittance. The impedance is obtained by inverting the admittance expression and separating the result into real and imaginary parts. The electrode impedance can now be calculated as a function of the frequency for a given set of rate constants and at a certain potential. These calculations are easily done on a personal computer, and by using the analytical solutions given previously the calculations can be performed much faster in comparison with the use of numerical solutions.

\section{References}

1. Macdonald, J. R. Impedance Spectroscopy, John Wiley \& Sons, New York 1987.

2. Gabrielli, C. Identification of Electrochemical Processes by Frequency Response Analysis, Monogr. No. 004/83, Schlumberger Instruments, Farnborough, UK 1980.

3. Ahlberg, E. and Anderson, H. Acta Chem. Scand. 46 (1992) 15.

4. Macdonald, D. D. and Urquidi-Macdonald, M. J. Electrochem. Soc. 132 (1985) 2316.

5. Macdonald, D. D. and Urquidi-Macdonald, M. J. Electrochem. Soc. 133 (1986) 2018. 
6. Macdonald, D. D. and Urquidi-Macdonald, M. J. Electrochem. Soc. 137 (1990) 515.

7. Urquidi-Macdonald, M., Real, S. and Macdonald, D. D. Electrochem. Acta 35 (1990) 1559.

8. Epelboin, I. and Keddam, M. J. Electrochemical Soc. 117 (1970) 1052.

9. Cao, C.-N. Electrochim. Acta 35 (1990) 831.

10. Cao, C.-N. Electrochim. Acta 35 (1990) 837.

11. Gaiser, L. and Heusler, K. E. Electrochim. Acta 15 (1970) 161

12. Hurlen, T. and Fischer, P. J. Electroanal. Chem. 61 (1975) 165

13. Heusler, K. E. Z. Elektrochem. 62 (1958) 582.

14. Cachet, C. and Wiart, R. J. Electroanal. Chem. 111 (1980) 235.

15. Cachet, C. and Wiart, R. J. Electroanal. Chem. 129 (1981) 103.
16. Deslouis, C., Duprat, M. and Tournillon, C. Corrosion Sci. 29 (1989) 13.

17. Armstrong, R. D. and Edmondson, K. Electrochem. Acta 18 (1973) 937.

18. Macdonald, J. R. Electrochim. Acta 35 (1990) 1483, and references therein.

19. Armstrong, R. D., Firman, R. E. and Thirsk, H. L. Faraday Discuss. Chem. Soc. 56 (1973) 244.

20. Gerischer, H. Z. Phy's. Chem. 158 (1951) 286.

21. Gerischer, H. Z. Phys. Chem. 206 (1952) 55.

22. Epelboin, I., Keddam, M. and Takenouti, H. J. Appl. Electrochem. 2 (1972) 71

23. Lorenz, W. J. and Mansfield, F. Corrosion Sci. 21 (1981) 647.

Received January 15, 1991. 